\title{
Weitere Beiträge zur Kenntnis der Hexahalogenotellureate.
}

\section{Von}

A. Gotbier und F. FluRy.

Mit 12 Figuren im Text.

In den letzten Jahren haben wir über Teile einer systematischen, die Halogenosalze des Tellurs betreffenden Untersuchung berichtet, ${ }^{1}$ die sich im Anschlusse an unsere früheren gemeinsamen Arbeiten über Tellur und an die Studien des einen von uns über die Platinmetalle als notwendig erwiesen hatten, und die auf unsere Veranlassung hin von den Herren H. Micheler ${ }^{2}$ und C. Ewald ${ }^{3}$ ausgefübrt worden waren. Wir hatten die Fortsetzung dieser Versuche in Aussicht gestellt und können nun heute eine neue Anzahl von Hexahalogenotellureaten beschreiben, die Herr V. WeinzIERL ${ }^{4}$ auf unsere Anregung hin bereitet und untersucht hat, so dab nunmehr eine - soweit es unsere eigenen Interessen berührt - lückenlose Reihe dieser Halogenosalze vorliegt.

Die Darstellung der weiter unten beschriebenen Anlagerungsverbindungen erfolgte im allgemeinen wie die der früher bereiteten so, da $B$ man eine bestimmte Menge der halogenwasserstoffsauren, ihrem Gehalte nach bekannten Lösung von gereinigtem Tellardioxyd, in welcher bekanntlich die Verbindungen $\mathrm{H}_{2} \mathrm{TeCl}_{6}$ bzw. $\mathrm{H}_{2} \mathrm{TeBr}_{6}$ enthalten sind, mit abgewogenen und in verdünnter Halogenwasserstoffsäure bzw. in Alkohol aufgelösten Mengen des betreffenden Am. moniumhalogenids bei gewöhnlicher Temperatur reagieren ließ. Die tellurhaltige Flüssigkeit war immer im Überschusse vorhanden, und andererseits wurde jederzeit dafür gesorgt, daB bei der Bildung der Reaktionsprodukte genügend viel Halogenwasserstoff zugegen war, der die gegebenenfalls eintretende Abscheidung von telluriger Säure sicher verhinderte. In vielen Fällen wurde die Anlagerungsverbin-

1 Journ. prakt. Chem. [2] 83 (1911), 145; 86 (1912), 150.

2 Dissertation, Erlangen 1911.

3 Dissertation, Erlangen 1912.

4 Betreffs aller Einzelheiten wird auf die demnächst erscheinende Dissertation des Herrn V. WelnziknL verwiesen. 
dung sogleich in Form eines kristallinischen Niederschlages abgeschieden; in anderen Fällen entstand zunächst ein Öl, das teilweise schon beim kräftigen Schütteln oder beim Reiben kristallinisch erstarrte oder aber beim Erwärmen nach Zugabe von Alkohol in Lösung ging und sich dann beim Erkalten der Filtrate kristallinisch abschied; wieder in anderen Fällen versagten auch diese Mittel; man mußte z. B. verschiedentlich starke Halogenwasserstoffsäure zusetzen, oder den zur Lösung der Ammoniumhalogenide benutzten Alkohol wegkochen, oder stärker verdünnte Lösungen anwenden und der freiwilligen Kristallisation überlassen usw. Wie man aus dieser kurzen Schilderung ersieht, war schon die erste Bereitung der Halogenosalze des öfteren mit großen Schwierigkeiten verbunden. Und abermals erschien es zunächst fast aussichtslos, die Präparate durch Umkristallisieren reinigen zu können. Da wieder die gleichen Beobachtungen wie früher gemacht wurden, haben wir auch diesmal ein Verfahren benutzen lassen, dessen sich der eine von uns bei seinen Untersuchungen über die Halogenosalze der Platinmetalle bei leicht zersetzlichen Präparaten oftmals mit Vorteil bedient hatte, und das darin besteht, daB man die erhaltenen Abscheidungen in Gegenwart der Bestandteile, also unter der Mutterlauge selbst, erhitzt und durch vorsichtigen Zusatz der entsprechenden verdünnten Halogenwasserstoffsäure, gegebenenfalls unter Zugabe einiger Tropfen Alkohol zur Lösung bringt und die Filtrate der freiwilligen Kristallisation überläbt, bzw. sie mit einem kleinen zurückbehaltenen Kriställchen impft.

Alle Verbindungen zeichnen sich durch lebhaften Glanz und prachtvolle Farbe aus. Die Chlorosalze sind gelb, lassen das Licht unter dem Mikroskop gelb durchscheinen und liefern beim Zerreiben gelbe Pulver. Die Bromosalze sind rot, scheinen rot durch und lassen sich zu roten Pulvern verreiben. Alle Präparate erweisen sich als wasserfrei. Sie entsprechen der allgemeinen Zusammensetzung $\left.\left[\mathrm{R} \cdot \mathrm{NH}_{3}\right]_{2} \mathrm{TeHal}\right]_{6}$, sind zum gröBten Teile an der Luft lange Zeit beständig und können im lufttrockenen Zustande analysiert werden.

Der kristallographischen Untersuchung - sie wurde von Herrn V. Weinzieri im mineralogisch-geologischen Institute der Technischen Hochschule Stuttgart ausgeführt, und wir sind dem Vorstande dieses lnstituts, Herrn Professor Dr. A. Sader für die gütige Unterstützung, die er unserem Mitarbeiter hat angedeihen lassen, zu Jehlaftem Danke verpflichtet - stellten sich ziemlich große Schwierig- 
keiten in den Weg. Abgesehen davon, daB kein Einbettungsmittel aufgefunden werden konnte, das die Präparate nicht auch chemisch angegriffen hätte, waren die Kristalle nur zum geringsten T'eile zur kristallographischen Untersuchung geeignet. Sie waren entweder zu klein und zu unregelmäBig ausgebildet, oder sie waren zu dichten Aggregaten verwachsen, an welchen man dann natürlich keine Flächen erkennen konnte. Nur diejenigen Kristalle, welche sich erst im Verlaufe von einigen Tagen ausgeschieden hatten, waren brauchbar, da sie meist eine Größe von $0.5 \mathrm{~mm}$ bis $1.5 \mathrm{~cm}$ aufwiesen und manchmal sogar modellartig ausgebildet waren; manche von diesen prächtigen Substanzen waren dann aber wieder undurchsichtig und erschwerten dadurch die optische Untersuchung oder machten sie unmöglich. In diesen Fällen konnte man sich vielfach dadurch helfen, daß man die Präparate nochmals auf dem Objektträger aus der entsprechenden Halogenwasserstoffsäure umkristallisierte. Es wurde erkannt, daß die meisten dieser Anlagerungsverbindungen dem rhombischen oder dem monoklinen Systeme angehören; als regulär wurden nur Tetramethylammoniumhexachloro- und hexabromotellureat erkannt, die durchweg die Kombination Würfel und Oktaeder aufweisen. Die einander entsprechenden Chloro- und Bromosalze kristallisieren vielfach im gleichen Systeme, doch wurden mit Ausnahme des oben erwähnten Falles niemals gleiche Kristallformen bei ihnen gefunden.

Das Verhalten der neuen Anlagerungsverbindungen Wasser gegenüber entspricht dem früher beschriebenen. Sie lösen sich in sehr geringen Mengen Wasser teils schon bei gewöhnlicher Temperatur, immer aber beim Erwärmen zu sehr schwach gelben, vielfach auch farblosen Flüssigkeiten auf und werden durch den geringsten Überschuß von Wasser schnell und vollständig unter Abscheidung von telluriger Säure zersetzt. Auch bei diesen Präparaten wurde wieder beobachtet, daß die Zerlegung der Chlorosalze durch Wasser mit gröBerer Geschwindigkeit vor sich geht, als diejenige der Bromoverbindungen.

Die Halogenosalze sind gegen einen Überschuß von verdünnter Halogenwasserstoffsäure ebenfalls sehr empfindlich und auch aus den dann durch Kochen konzentrierten Lösungen scheiden sie sich nicht unzersetzt wieder aus. Man erkennt das schon leicht an der viel helleren Farbe dieser neuen Abscheidungen, besonders deutlich aber daran, daß die Präparate schon beim Abfiltrieren trübe werden, sich nach kurzem Aufbewahren an der Luft mit einem weißen 
Überzug von telluriger Säure bedecken und nach wenigen Stunden vollständig zersetzt sind.

$96 \%$ iger Alkohol bewirkt bei gewöhnlicher Temperatur sehr weitgehende Zersetzung der Halogenosalze unter Abscheidung von telluriger Säure; bei Siedehitze entstehen mit wenig Alkohol farblose bzw. ganz schwach gelbe, klare Lösungen.

\section{Experimenteller 'Teil.}

(Bearbeitet von Victor Weinziera.)

Die Ausgangsmaterialien waren die gleichen wie bei den früheren Versuchen. ${ }^{\top}$ Die Ammoniumhalogenide wurden teils als solche von C. A. F. Kahlbaum-Berlin bezogen, teils aus den entsprechenden Aminen, die dieselbe Firma geliefert hatte, selbst bereitet.

An analytischen Methoden wurden benutzt: zur gewichtsanalytischen Bestimmung des Tellurs das von VICTOR LenHer und A. W. Hombergeri ${ }^{2}$ ausgearbeitete Verfahren und zur titrimetrischen Halogenbestimmung die Methode von VidTor Lenher. ${ }^{3}$

\section{A. Chlorosalze.}

\section{Tetramethylammoniumhexachlorotellureat, $\left[\left(\mathrm{CH}_{3}\right)_{4} \cdot \mathrm{N}\right]_{2} \mathrm{TeCl}_{6}$.}

Die Verbindung läBt sich nur in Gegenwart von konzentrierter Salzsäure sogleich als gelber kristallinischer Niederschlag fälllen und stellt im gereinigten Zustande gut ausgebildete Kuboktaeder dar. Farbe: 9 Gelb $r^{4}$ Ist an der Luft sehr beständig.

$0.2826 \mathrm{~g}$ Substanz verbrauchten: $34.45 \mathrm{ccm} 1 / 10^{\text {-norm. }} \mathrm{AgNO}_{3}$. Berechnet für $\mathrm{C}_{8} \mathrm{H}_{24} \mathrm{~N}_{2} \mathrm{TeCl}_{6}$ : Cl $43.56 \%$ Gefunden: $43.22 \%$

\section{Tripropylammoniumhexachlorotellureat, $\left[\left(\mathrm{C}_{3} \mathrm{H}_{7}\right)_{3} \cdot \mathrm{NH}_{2}\right]_{2} \mathrm{TeCl}_{6}$.}

Scheidet sich beim Zusammenbringen der Komponenten sogleich als gelber, aus prächtig glänzenden, derben Kriställchen bestehender Niederschlag ab. Man erkennt unter dem Mikroskop ziemlich schmale, meist von Basis und Pyramide begrenzte rhombische Prismen von

1 Journ. pratit. Chem. [2] $\$ 3$ (1911), 149.

a Journ. Amer. Chem. Soe. 30 (1908), 387.

"Journ. Amer. Chem. Soc. 22 (1900), 136.

+ Alle Farben sind nach RadDe bestimmt worden. 
mittlerer Licht- und Doppelbrechung. Farbe: 9 Gelb $s$. Die Präparate zeigten beim Aufbewahren an der Luft keinerlei Zersetzungserscheinungen.

$0.2200 \mathrm{~g}$ Substanz verbrauchten: $20.85 \mathrm{ccm} 1 / 10^{- \text {norm. }} \mathrm{AgNO}_{3}$. Berechnet für $\mathrm{C}_{18} \mathrm{H}_{44} \mathrm{~N}_{2}{ } \mathrm{TeCl}_{6}$ :

Cl $33.85 \%$ Gefunden: $33.60 \%$

3. Di-i-butylammoniumhexachlorotellureat, $\left[\left(\mathrm{C}_{4} \mathrm{H}_{9}\right)_{2} \cdot \mathrm{NH}_{2}\right]_{2} \mathrm{TeCl}_{6}$.

Läßt sich nur aus sehr stark konzentrierten Lösungen der Bestandteile in Form ganz unregelmäBig ausgebildeter, an der Luft bald zerfließender Kristalle gewinnen. Farbe: 10 Gelbgrün $t$.

$0.1050 \mathrm{~g}$ Substanz verbrauchten: $10.3 \mathrm{ccm} \mathrm{1/10}$-norm. $\mathrm{AgNO}_{3}$.

Berechnet für $\mathrm{C}_{16} \mathrm{H}_{40} \mathrm{~N}_{2} \mathrm{TeCl}_{6}$ :

Cl $35.42 \%$

Gefunden:

$34.78 \%$

4. Tri-i-butylammoniumhexachlorotellureat, $\left[\left(\mathrm{C}_{4} \mathrm{H}_{9}\right)_{3} \cdot \mathrm{NH}\right]_{2} \mathrm{TeCl}_{6}$.

Das Halogenosalz setzt sich beim Vermischen der Komponenten zunächst immer als gelbes Öl ab, erstarrt aber ziemlich schnell kristallinisch. Man erhält bei der Reinigung schön glänzende, rein gelbe Nädelchen, die sich unter dem Mikroskop als Aggregate von schwach lichtbrechenden Kriställchen erkennen lieBen. Aus der Mutterlauge schieden sich nach einigen Tagen noch größere Kristalle aus, welche aus nahezu modellartig ausgebildeten, tetragonalen, meist von der Basis abgestumpften Pyramiden bestanden. Farbe: 8 Gelb $r$. Die Präparate sind durchaus luftbeständig.

$0.1978 \mathrm{~g}$ Substanz verbrauchten: $16.5 \mathrm{ccm} 1 / 10^{\text {-norm. }} \mathrm{AgNO}_{3}$.

Berechnet für $\mathrm{C}_{24} \mathrm{H}_{56} \mathrm{~N}_{2} \mathrm{TeCl}_{6}$ :

Cl $29.83 \%$

Gefunden:

$29.57 \%$

5. Allylammoniumhexachlorotellureat, $\left[\mathrm{C}_{3} \mathrm{H}_{5} \cdot \mathrm{NH}_{3}\right]_{2} \mathrm{TeCl}_{6}$.

Setzte sich nach einiger Zeit in derben gelben Kristallen $a b$, die beim Umkristallisieren in Form von gelben Blättchen zurückgewonnen werden. Die Produkte sind durch verhältnismäBig geringe Beständigkeit ausgezeichnet. Sie zersetzen sich an der Luft schnell und selbst im geschlossenen Gläschen, indem sie farblos und durchsichtig werden, behalten dabei aber ihre rechteckige bis quadratische Form bei.

$0.0436 \mathrm{~g}$ Substanz verbrauchten: $5.65 \mathrm{ccm} 1 / 10^{- \text {norm. }} \mathrm{AgNO}_{3}$. 
Berechnet für $\mathrm{C}_{6} \mathrm{H}_{16} \mathrm{~N}_{2} \mathrm{TeCl}_{6}$ :

Gefunden:

Cl $46.62 \%$

$45.94 \%$

6. Tri-i-amylammoniumbexachlorotellureat, $\left[\left(\mathrm{C}_{5} \mathrm{H}_{11}\right)_{3} \cdot \mathrm{NH}\right]_{2} \mathrm{TeCl}_{6}$.

Die gelösten Bestandteile liefern bei der Vereinigung eine ölige Abscheidung, die ziemlich schnell kristallinisch wird und nach der üblichen Reinigung in hellgelben, glänzenden Kriställchen vorliegt. Sie gehören dem monoklinen System an, sind von dem Quer- und Vertikalprisma und der Querfäche begrenzt (Fig. 1) und zeigen starke Licht- und Doppelbrechung. Farbe: 11 Gelbgrün n. Beim

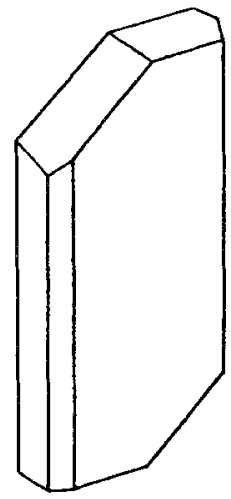

Fig. 1.

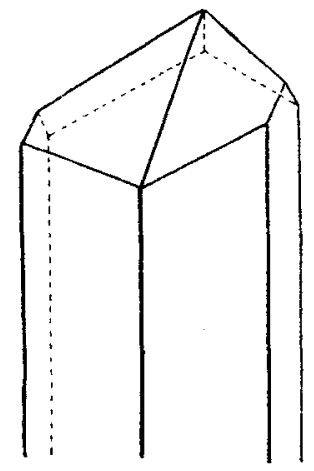

Fig. 2.

Aufbewahren der Substanzen an der Luft wurden keine Zersetzungserscheinungen beobachtet.

$0.1322 \mathrm{~g}$ Substanz verbrauchten: $9.85 \mathrm{ccm} 1 / 10^{- \text {norm. }} \mathrm{AgNO}_{3}$.

Berechnet für $\mathrm{C}_{30} \mathrm{H}_{68} \mathrm{~N}_{2} \mathrm{TeCl}_{6}$ :

$$
\text { Cl } 26.70 \%
$$

Gefunden:

$26.42 \%$

7. Guanidiniumbexachlorotellureat, $\left[\mathrm{HN}: \mathrm{C} .\left(\mathrm{NH}_{2}\right)_{2} \cdot \mathrm{H}_{2} \mathrm{TeCl}_{6}\right.$.

Die Anlagerungsverbindung setzt unter gewöhnlichen Konzentrationsverhältnissen erst nach einiger Zeit, aus stärker konzentrierten Lösungen sogleich gelbe derbe Nadeln ab, welche rhombische, meist von der Basis abgestumpfte Pyramiden darstellen und sehr starke Licht- und Doppelbrechung aufweisen. Farbe: 10 Gelbgrün $r$. Die Präparate zersetzen sich an der Luft innerhalb einiger Tage, indem sie oberflächlich braun werden.

0.2240 g Substanz verbrauchten: $29.0 \mathrm{ccm} 1 / 10^{-n o r m} . \mathrm{AgNO}_{3}$.

Berechnet für $\mathrm{C}_{2} \mathrm{H}_{12} \mathrm{~N}_{6} \mathrm{TeCl}_{6}$ :

Gefunden:

Cl $46.21 \%$

$45.90 \%$. 


\section{8. m-Chloraniliniumhexachlorotellureat, $\left[\mathrm{C}_{6} \mathrm{H}_{4} \cdot \mathrm{Cl} \cdot \mathrm{NH}_{3}\right]_{2} \mathrm{TeCl}_{6}$.}

Kristallisiert erst nach einiger Zeit aus und liegt nach der Reinigung in zarten und glänzenden, bräunlich-gelben, monoklinen Kristallen von mittlerer Lichtbrechung und sehr starker Doppelbrechung vor. An Flächen (Fig. 2) treten allgemein auf: das Vertikalprisma, die Längsfläche und die Pyramide; die Auslöschungsschiefe beträgt $25^{\circ}$. Farbe: 8 Gelb $s$. Die Kristalle färben sich beim Aufbewahren an der Luft bald tiefer braun.

$0.2662 \mathrm{~g}$ Substanz verbrauchten: $26.30 \mathrm{ccm} 1 / 10$-norm. $\mathrm{AgNO}_{3}$.

Berechnet für $\mathrm{C}_{12} \mathrm{H}_{14} \mathrm{Cl}_{2} \mathrm{~N}_{2} \mathrm{TeCl}_{8}$ : Cl $35.62 \%$

Gefunden: $35.03 \%$.

\section{9. p-Chloraniliniumhexachlorotellureat, $\left[\mathrm{C}_{8} \mathrm{H}_{4} \cdot \mathrm{Cl} \cdot \mathrm{NH}_{3}\right]_{2} \mathrm{TeCl}_{6}$.}

Auch hier tritt kristallinische Abscheidung aus den gemischten Lösungen der Bestand. teile erst nach einiger Zeit ein. Man gewann schön gelbe, bis $\mathrm{zu} 5 \mathrm{~cm}$ lange und $4 \mathrm{~mm}$ breite, flache Nadeln, die sich als monoklin erwiesen und (Fig. 3) die Flächen $O P, P \infty$, $\infty P_{\infty}$ und $\infty P_{\infty}$ aufwiesen. Farbe: $10 \mathrm{Gelb}$ grün $r$. Die Kristalle halten sich an der Luft eine Zeit lang, beginnen aber nach etwa 3 Wochen sich oberflächlich zu bräunen.

$0.1312 \mathrm{~g}$ Substanz verbrauchten: $13.25 \mathrm{ccm}$

$$
1 / 10 \text {-norm. } \mathrm{AgNO}_{3} \text {. }
$$

Berechnet für $\mathrm{C}_{12} \mathrm{H}_{14} \mathrm{Cl}_{2} \mathrm{~N}_{2} \mathrm{TeCl}_{6}$ :

$$
\text { Cl } 35.62 \%
$$

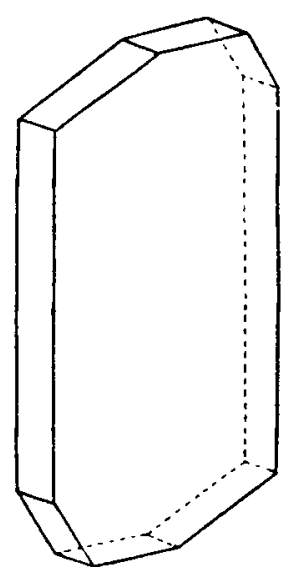

Fig. 3.

Gefunden:

$35.81 \%$.

10. i-Amylaniliniumhexachlorotellureat, $\left[\mathrm{C}_{6} \mathrm{H}_{5} \cdot \mathrm{NH}_{2} \cdot \mathrm{C}_{5} \mathrm{H}_{11}\right]_{2} \mathrm{TeCl}_{6}$.

Die gelösten Bestandteile liefern beim Vermischen eine gelbe, ölige Abscheidung, die groBe Neigung zeigt, in diesem Zustande zu verharren. Gibt man bei der Darstellung des Halogenosalzes etwas mehr Alkohol, als zur Klärung der Flüssigkeit nötig ist, hinzu und unterstützt man gleichzeitig diesen LösungsprozeB durch schwaches Erwärmen, so erscheinen aus der klaren Flüssigkeit nach einiger Zeit gelbe Kriställchen, die abfiltriert und an der Luft aufbewahrt, sich innerhalb weniger Stunden vollständig zersetzen, indem sie 
wieder ölig zerfließen. Man gewann nach vielen Versuchen schließ. lich ein haltbares Präparat dadurch, daß man jegliche Temperaturerhöhung vermied, in Form von rhombischen, sehr einfach ausgebildeten, fast nur ron $\infty P$ und $P$ begrenzten Kristallen. Farbe: 10 Gelbgrün $t$.

$0.1848 \mathrm{~g}$ Substanz verbrauchten: $16.45 \mathrm{ccm} 1 / 10^{-n o r m} . \mathrm{AgNO}_{3}$.

Berechnet für $\mathrm{C}_{22} \mathrm{H}_{36} \mathrm{~N}_{2} \mathrm{TeCl}_{6}$ :

Cl $31.82 \%$

Gefunden:

$31.55 \%$.

11. Benzalaniliniumhexachlorotellureat, $\left[\mathrm{C}_{8} \mathrm{H}_{5} . \mathrm{CH}: \mathbf{N} . \mathrm{C}_{6} \mathrm{H}_{5} . \mathrm{H}_{2}\right]_{2} \mathrm{TeCl}_{6}$. Aus den vereinigten Lösungen der Komponenten setzen sich nach einiger Zeit schön gelbe, sehr feine Nädelchen ab, die sich, wenn man sie längere Zeit unter der Mutterlauge liegen läßt, in ziemlich große, glänzende Blättchen verwandeln. Sie weisen sehr schwache Licht- und Doppelbrechung auf, löschen schief aus und sind wahrscheinlich monoklin. Farbe: 10 Gelbgrün $q$. Die Kristalle verlieren nach mehrtägigem Liegen an der Luft ihren Glanz, werden undurchsichtig und färben sich dann grün.

$0.2322 \mathrm{~g}$ Substanz verbrauchten: $19.95 \mathrm{ccm} 1 / 10$-norm. $\mathrm{AgNO}_{3}$. Berechnet für $\mathrm{C}_{26} \mathrm{H}_{14} \mathrm{~N}_{2} \mathrm{TeCl}_{6}$ :

Cl $30.64 \%$ Gefunden: $30.46 \%$

12. Äthylbenzylaniliniumhexachlorotellureat, $\left[\mathrm{C}_{6} \mathrm{H}_{5} \cdot \mathrm{NH} \cdot \mathrm{C}_{2} \mathrm{H}_{5} \cdot \mathrm{C}_{6} \mathrm{H}_{5} \cdot \mathrm{CH}_{2}\right]_{2} \mathrm{TeCl}_{6}$.

Scheidet sich als $\ddot{\text { Ol }}$ aus und erstarrt nach und nach kristallinisch. Reinigt man das Produkt dann unter Zusatz von Alkohol, so gewinnt man prachtroll glänzende, rein gelbe, monokline Kriställchen, die mittlere Licht- und Doppelbrechung, sowie eine Auslöschungsschiefe von etwa $50^{\circ}$ aufweisen. Farbe: 8 Gelb $s$. Zersetzungserscheinungen wurden an einem Präparate, das man wochenlang an der Luft liegen lieB, nicht beobachtet.

0.2018 g Substanz verbrauchten: $15.45 \mathrm{ccm} 1 / 10^{- \text {norm. }} \mathrm{AgNO}_{3}$.

Berechnet für $\mathrm{C}_{30} \mathrm{H}_{36} \mathrm{~N}_{2} \mathrm{TeCl}_{6}$ : Gefunden:

Cl $27.83 \%$ $27.15 \%$.

13. Dimethyl-o-Toluidiniumhexachlorotellureat, $\left[\mathrm{C}_{6} \mathrm{H}_{4} \cdot \mathrm{CH}_{3} \cdot \mathrm{N} \cdot\left(\mathrm{CH}_{3}\right)_{2} \cdot \mathrm{H}\right]_{2} \mathrm{TeCl}_{6}$.

Erscheint zunächst als gelbes Öl, das nach einiger Zeit von selbst, beim Reiben oder Schütteln fast augenblicklich erstarrt. Bei 
der Reinigung, und zwar unter Zusatz einiger Tropfen Alkohol, erhält man sehr kleine, glänzende gelbe Nädelchen. Aus deren Mutterlauge setzten sich nach mehreren Tagen gröBere Kristalle ab, die, sehr gut ausgebildet, sich als dem rhombischen Systeme zugehörig erkennen ließen; sie sind fast allgemein begrenzt von dem Vertikalprisma, dem Brachypinakoid, dem Makropinakoid, dem Makrodoma, einer Pyramide, dem Brachydoma und der Basis

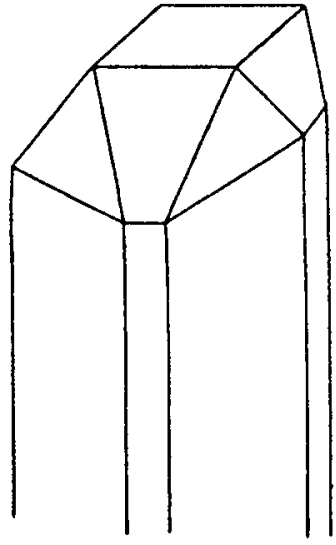

Fig. 4 . (Fig. 4). Farbe: 9 Gelb s. Die Kristalle verändern sich an der Luft nicht.

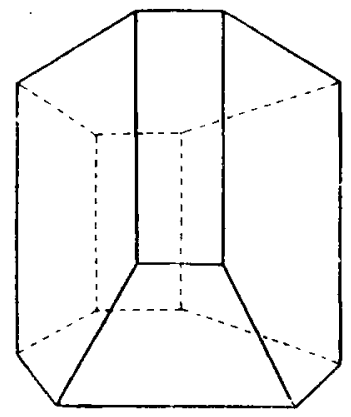

Fig. 5 .

$0.2718 \mathrm{~g}$ Substanz verbrauchten: $26.45 \mathrm{ccm} 1 / 10^{-}$norm. $\mathrm{AgNO}_{3}$.

Berechnet für $\mathrm{C}_{18} \mathrm{H}_{28} \mathrm{~N}_{2} \mathrm{TeCl}_{6}$ :

Gefunden:

$\mathrm{Cl} 34.74 \%$

$34.50 \%$

14. Dimethyl-p-Toluidiniumhexachlorotellureat, $\left[\mathrm{C}_{6} \mathrm{H}_{4} \cdot \mathrm{CH}_{3} \cdot \mathbf{N} \cdot\left(\mathrm{CH}_{3}\right)_{2} \cdot \mathrm{H}\right]_{2} \mathrm{TeCl}_{8}$.

Auch dieses Halogenosalz fällt zunächst als öl aus. Erwärmt man es mit der Mutterlauge unter Zusatz von etwas Alkohol, so erhält man eine klare Lösung, aus der bei schnellem Abkühlen wiederum ein Öl, beim langsamen Erkalten dagegen feine gelbe Nädelchen erhalten werden. Im Filtrate dieses Niederschlags fand man nach Verlauf von mehreren Tagen derbe, stark gedrungene und ziemlich dunkle Kristalle vor, die sehr gut ausgebildet sind, dem rhombischen System angehören und an Flächen $\infty P, P \infty$, $\infty P_{\infty}$ und $O P$ aufweisen (Fig. 5). Farbe: 10 Gelbgrün $r$. Die Kristalle büßen auch bei längerem Aufbewahren an der Luft nichts von ihrem Glanze ein.

$0.1130 \mathrm{~g}$ Substanz verbrauchten: $11.1 \mathrm{ccm} 1 / 10^{-n o r m} . \mathrm{AgNO}_{3}$. 
Berechnet für $\mathrm{C}_{18} \mathrm{H}_{28} \mathrm{~N}_{2} \mathrm{TeCl}_{6}$ :

Gefunden:

Cl $34.74 \%$ $34.83 \%$

15. Psendocumidiniumhexachlorotellureat, $\left[\mathrm{C}_{6} \mathrm{H}_{2} \cdot\left(\mathrm{CH}_{3}\right)_{3} \cdot \mathrm{NH}_{3}\right]_{2} \mathrm{TeCl}_{6}$.

Die Anlagerungsverbindung ist nur zu erhalten, wenn man in stark salzsaurer Lösung arbeitet und das Reaktionsgemisch vorsichtig noch etwas einengt. Unter diesen Bedingungen scheiden sich gelbe Kristalle $a b$, die mittlere Licht- und Doppelbrechung aufweisen, schief auslöschen und wahrscheinlich monoklin sind. Farbe: 8 Gelb $s$. Die Kristalle zersetzen sich an der Luft nach einigen Tagen.

0.0932 g Substanz verbrauchten: $9.05 \mathrm{ccm}{ }^{1} / 10$-norm. $\mathrm{AgNO}_{3}$.

Berechnet für $\mathrm{C}_{18} \mathrm{H}_{28} \mathrm{~N}_{2} \mathrm{TeCl}_{6}$ :

Gefunden:

Cl $34.74 \%$

$34.43 \%$

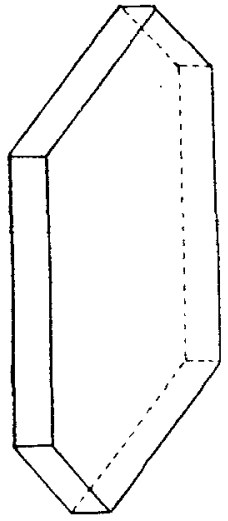

Fig. 6.

\section{6. $\beta$-Picoliniumhexachlorotellureat,}

$\left[\mathrm{C}_{5} \mathrm{H}_{4} \cdot \mathrm{CH}_{3} \cdot \mathrm{NH}_{2}\right]_{2} \mathrm{TeCl}_{6}$.

Bildet sich sogleich als gelber kristallinischer Niederschlag und stellt gereinigt prachtvoll gelbe Blättchen mit schwacher lichtbrechung und mittlerer Doppelbrechung, sowie von einer Auslöschungsschiefe von etwa $60^{\circ}$ dar. Sie sind monoklin (Fig. 6). Farbe: 10 Gelbgrün $m$. Die Kristalle hatten auch nach vierwöchentlichem Aufbewahren an der Luft nicht an ihrer Klarheit verloren.

$0.0943 \mathrm{~g}$ Substanz verbrauchten: $10.7 \mathrm{ccm}$ $1 / 10^{- \text {norm. }} \mathrm{AgNO}_{3}$.

Berechnet für $\mathrm{C}_{12} \mathrm{H}_{16} \mathrm{~N}_{2} \mathrm{TeCl}_{6}$ :

Gefunden:

Cl $40.26 \%$ $41.17 \%$

17. Lutidiniumhexachlorotellureat, $\left[\mathrm{C}_{5} \mathrm{H}_{3} \cdot\left(\mathrm{CH}_{3}\right)_{2} \cdot \mathrm{NH}\right]_{2} \mathrm{TeCl}_{6} \cdot{ }^{1}$

Das beim Vermischen der Bestandteile sich sogleich ausscheidende Halogenosalz wird beim Umkristallisieren in gelblich grünen Nädelchen zurückgewonnen. Man erkennt unter dem Mikroskope sehr stark licht- und doppelbrechende, einfache, rhombische Prismen. Farbe: 12 Gelbgrün $s$. Die Präparate sind an der Luft durchaus beständig.

$0.1108 \mathrm{~g}$ Substanz verbrauchten: $11.9 \mathrm{~cm}{ }^{1} / 10^{-n o r m} . \mathrm{AgNO}_{3}$.

1 Ausgangsmaterial: Lutidin känflich. 
Berechnet für $\mathrm{C}_{14} \mathrm{H}_{20} \mathrm{~N}_{2} \mathrm{TeCl}_{6}$ :

$$
\text { Cl } 38.24 \%
$$

Gefunden: $38.08 \%$.

18. Kollidiniumhesachlorotellureat, $\left[\mathrm{C}_{5} \mathrm{H}_{2} \cdot\left(\mathrm{CH}_{3}\right)_{3} \cdot \mathrm{NH}\right]_{2} \mathrm{TeCl}_{6}{ }^{1}$

Scheidet sich erst nach einiger Zeit in sehr gut und regelmäBig ausgebildeten, tetragonalen Kristallen aus, die meist von dem Protound Deuteroprisma, sowie von der Pyramide erster und zweiter Art begrenzt sind; gewöhnlich ist noch die Basis vorhanden, die dann die Pyramiden abstumpft. Farbe: 8 Gelb $s$. Verändert sich beim Aufbewahren an der Luft nicht.

$0.2820 \mathrm{~g}$ Substanz verbrauchten: $29.0 \mathrm{ccm}^{1} / 10^{-n o r m} . \mathrm{AgNO}_{3}$.

Berechnet für $\mathrm{C}_{16} \mathrm{H}_{24} \mathrm{~N}_{2} \mathrm{TeCl}_{8}$ :

Gefunden:

Cl $36.40 \%$

$36.46 \%$

19. Piperidiniumhexachlorotellureat, $\left[\mathrm{C}_{5} \mathrm{H}_{10} \cdot \mathrm{NH}_{2}\right]_{2} \mathrm{TeCl}_{6}$.

Fällt erst nacb einiger Zeit aus und bildet leuchtend gelbe, stark glänzende, rhombische Kristalle. Neben flächenreicheren, dann allerdings undeutlich ausgebildeten Individuen finden sich verhältnismäBig viele, nur aus $O P$ und $\infty P$ bestehende. Farbe: 8 Gelb $r$. Die schwach licht- und doppelbrechenden Kristalle können ziemlich lange an der Luft aufbewahrt werden, ohne sich zu zersetzen.

$0.2183 \mathrm{~g}$ Substanz verbrauchten: $25.2 \mathrm{ccm} 1 / 10^{\text {-norm. }} \mathrm{AgNO}_{3}$.

Berechnet für $\mathrm{C}_{10} \mathrm{H}_{24} \mathrm{~N}_{2} \mathrm{TeCl}_{6}$ :

Gefunden:

Cl $41.52 \%$

$40.93 \%$

\section{0. i-Chinoliniumhexachlorotellureat, $\left[\mathrm{C}_{9} \mathrm{H}_{7} \cdot \mathrm{NH}\right]_{2} \mathrm{TeCl}_{6}$.}

Das Halogenosalz wird beim Zusammenbringen der gelösten Bestandteile sogleich als hellgelber, kristallinischer Niederschlag ausgeschieden, der nach der Reinigung in prachtroll seideglänzenden Nadeln vorliegt. Man erkennt unter dem Mikroskope monokline Prismen von mittlerer Licht- und Doppelbrechung und einer Auslöschungsschiefe von etwa $20^{\circ}$. Farbe: 9 Gelb $s$. Die Präparate behalten ihren Glanz auch bei längerem Liegen an der Luft bei.

$0.3801 \mathrm{~g}$ Substanz verbrauchten: $37.6 \mathrm{~cm} \mathrm{1/10}$-norm. $\mathrm{AgNO}_{3}$.

Berechnet für $\mathrm{C}_{18} \mathrm{H}_{18} \mathrm{~N}_{2} \mathrm{TeCl}_{6}$ :

Gefunden:

Cl $35.44 \%$ $35.07 \%$

1 Ausgangsmaterial: Kollidin aus Aldehyd. 


\section{Benzylmethylammoniumhexachlorotellureat, $\left[\mathrm{C}_{6} \mathrm{H}_{5} \cdot \mathrm{CH}_{2} \cdot \mathrm{NH}_{2} \cdot \mathrm{CH}_{3}\right]_{2} \mathrm{TeCl}_{6}$.}

Wird sogleich kristallinisch gefällt und bildet in reinem $\mathrm{Zu}$ stande ziemlich grobe und lebhaft seideglänzende, gelbe, wahrscheinlich monokline Nadeln. Sie weisen mittlere Lichtbrechung, verhältnismäßig starke Doppelbrechung und eine Auslöschungsschiefe von etwa $5^{0}$ auf. Farbe: 10 Gelbgrün $r$. An der Luft sehr beständig.

$0.1456 \mathrm{~g}$ Substanz verbrauchten: $14.85 \mathrm{ccm} 1 / 10^{- \text {norm. }} \mathrm{AgNO}_{3}$.
Berechnet für $\mathrm{C}_{16} \mathrm{H}_{24} \mathrm{~N}_{2} \mathrm{TeCl}_{6}$ : Gefunden:
Cl $36.40 \% \quad 36.16 \%$

22. Benzalmethylammoniumhexachlorotellureat, $\left[\mathrm{C}_{6} \mathrm{H}_{5} . \mathrm{CH}: \mathrm{NH} . \mathrm{CH}_{3}\right]_{2} \mathrm{TeCl}_{6}$.

Scheidet sich ebenfalls sofort kristallinisch ab und wird bei der Reinigung, die man vorteilhaft unter Zugabe von etwas Alkohol vornimmt, in Form von hellgelben, feinen Nädelchen gewonnen. Diese stellen meist gut ausgebildete rhombische Prismen von mittlerer Lichtbrechung und ziemlich starker Doppelbrechung dar; fast durchgängig wird die Kombination $\infty P, P \bar{\infty}, \infty P_{\infty}$ beobachtet. Farbe: 9 Gelb s. Die Präparate erwiesen sich als luftbeständig.

$0.2286 \mathrm{~g}$ Substanz verbrauchten: $23.4 \mathrm{ccm} 1 / 10^{\text {-norm. }} \mathrm{AgNO}_{3}$.

Berechnet für $\mathrm{C}_{16} \mathrm{H}_{20} \mathrm{~N}_{2} \mathrm{TeCl}_{6}$ : Gefunden: Cl $36.65 \% \quad 36.29 \%$

\section{Benzaläthylammoniumhexachlorotellureat,} $\left[\mathrm{C}_{6} \mathrm{H}_{5} \cdot \mathrm{CH}: \mathrm{NH} \cdot \mathrm{C}_{2} \mathrm{H}_{5}\right]_{2} \mathrm{TeCl}_{6}$.

Die Verbindung fällt zunächst als gelbes Öl aus, das nach und nach kristallinisch erstarrt. Löst man das Produkt dann unter $\mathrm{Zu}$ satz von Alkohol, so erscheinen prachtvoll glänzende, gelbe Kriställchen. Sie sind Aggregate von kleinen, faserigen, wahrscheinlich monoklinen Prismen, die mittlere Lichtbrechung, starke Doppelbrechung und eine Auslöschungsschiefe ron etwa $16^{\circ}$ aufweisen. Farbe: 8 Gelb $p$. Die Substanz lieB sich unverändert an der Luft aufbewahren.

$0.1674 \mathrm{~g}$ Substanz verbrauchten: $16.45 \mathrm{ccm} 1 / 10^{-n o r m}$. $\mathrm{AgNO}_{3}$.

Berechnet für $\mathrm{C}_{18} \mathrm{H}_{24} \mathrm{~N}_{2} \mathrm{TeCl}_{6}$ :

Gefunden:

Cl $34.97 \%$

$34.84 \%$. 


\section{B. Bromosalze.}

\section{Tetramethylammoniumhexabromotellureat, $\left[\left(\mathrm{CH}_{3}\right)_{4} \cdot \mathrm{N}\right]_{2} \mathrm{TeBr}_{6}$.}

Aus den gemischten Lösungen der Komponenten kristallisiert sofort ein Niederschlag aus, der gereinigt kleine, glänzende orangefarbige Kriställchen darstellt. Es sind reguläre, nahezu modellartig ausgebildete Kuboktaeder. Farbe: 4 Orange $o$. Die Präparate behielten während vierwöchentlichen Aufbewahrens an der Luft ihren Glanz bei.

$0.0168 \mathrm{~g}$ Substanz verbrauchten: $1.35 \mathrm{ccm} 1 / 10^{\text {-norm. }} \mathrm{AgNO}_{3}$.

$$
\text { Berechnet für } \mathrm{C}_{8} \mathrm{H}_{24} \mathrm{~N}_{2} \mathrm{TeBr}_{6} \text { : Gefunden: }
$$

$$
\text { Br } 63.49 \%
$$

2. Tripropylammoniumbexabromotellureat, $\left[\left(\mathrm{C}_{3} \mathrm{H}_{7}\right)_{3} \cdot \mathrm{NH}\right]_{2} \mathrm{TeBr}_{6}$.

Wird sogleich kristallinisch gefällt und liefert bei der Reinigung schön glänzende, rotgelbe Kriställchen. Es sind meist einfache, von der Basis abgestumpfte, rhombische Pyramiden von sehr starker Licht- und Doppelbrechung. Bemerkenswert ist, daB die einzelnen Kriställchen miteinander meist zu Ketten verwachsen sind. Farbe: 3 Zinnober $p$. Die Präparate waren an der Luft beständig.

$0.0832 \mathrm{~g}$ Substanz verbrauchen: $5.55 \mathrm{ccm} 3 / 10^{-n o r m} . \mathrm{AgNO}_{3}$.

Berechnet für $\mathrm{C}_{18} \mathrm{H}_{44} \mathrm{~N}_{2} \mathrm{TeBr}_{6}$ :

Gefunden:

$\operatorname{Br} 53.55 \%$

3. Di-i-butylammonium hexabromotellureat, $\left[\left(\mathrm{C}_{4} \mathrm{H}_{9}\right)_{2} \cdot \mathrm{NH}_{2}\right]_{2} \mathrm{TeBr}_{6}$.

Die Komponenten vereinigen sich zu einer orangefarbenen kristallinischen Abscheidung, die beim Umkristallisieren in wohlausgebildeten, rhombischen, von dem Vertikalprisma der Querfläche, dem Querprisma und einer Pyramide begrenzten Prismen von schwacher Licht- und Doppelbrechung zurückgewonnen werden. Farbe: 4 Orange $o$. Die Kristalle waren im Gegensatze zu denjenigen der entsprechenden Chloroverbindung luftbeständig.

$0.0180 \mathrm{~g}$ Substanz verbrauchten: $1.25 \mathrm{ccm} 1 / 10^{-n o r m} . \mathrm{AgNO}_{3}$.

Berechnet für $\mathrm{C}_{16} \mathrm{H}_{40} \mathrm{~N}_{2} \mathrm{TeBr}_{6}$ : Br $55.29 \%$ Gefunden: $55.50 \%$

4. Tri-i-butylammoniumhexabromotellureat, $\left[\left(\mathrm{C}_{4} \mathrm{H}_{9}\right)_{3} \cdot \mathrm{NH}\right]_{2} \mathrm{TeBr}_{6}$.

Beim Vermischen der gelösten Bestandteile tritt eine ölige $\mathrm{Ab}$ scheidung auf, die bald kristallisiert und, unter Zusatz von etwas 
Alkohol gereinigt, in glitzernden orangefarbigen Nädelchen und Blättchen wiedergewonnen wird. Die Präparate weisen schwache Licht- und Doppelbrechung auf und sind wahrscheinlich rhombisch. Farbe: 5 Orange $q$. Zersetzung der Substanz an der Luft wurde nicht beobachtet.

$0.2862 \mathrm{~g}$ Substanz verbrauchten: $17.6 \mathrm{ccm} 1 / 10^{-n o r m} . \mathrm{AgNO}_{3}$. Berechnet für $\mathrm{C}_{24} \mathrm{H}_{56} \mathrm{~N}_{2} \mathrm{TeBr}_{6}$ : Gefunden:

$$
\operatorname{Br} 48.93 \%
$$

5. Allylammoniumhexabromotellureat, $\left[\left(\mathrm{C}_{3} \mathrm{H}_{5} \cdot \mathrm{NH}_{3}\right)_{2} \mathbf{T e B r}_{6}\right.$.

Die Anlagerungsverbindung setzt sich sogleich als reichlicher, orangefarbiger, kristallinischer Niederschlag ab, und bildet nach der Reinigung gut ausgebildete, bis $6 \mathrm{~cm}$ lange und $1 \mathrm{~cm}$ breite, übereinandergelagerte und vielfach zu Rosetten verwachsene rote, monokline Kristalle. Farbe: 1 Zinnober $k$. Die Kristalle verlieren bei längerem Liegen an der Luft ihren Glanz, sind aber bedeutend beständiger als die der Chloroverbindung.

$0.2406 \mathrm{~g}$ Substanz verbrauchten: $19.95 \mathrm{ccm} 1 / 10^{\text {-norm. }} \mathrm{AgNO}_{3}$. Berechret für $\mathrm{C}_{6} \mathrm{H}_{16} \mathrm{~N}_{2} \mathrm{TeBr}_{6}$ : $\operatorname{Br} 66.31 \%$ Gefunden: $66.27 \%$

6. i-Amylammoniumhexabromotellureat, $\left[\mathrm{C}_{5} \mathrm{H}_{11} \cdot \mathrm{NH}_{3}\right)_{2} \mathrm{TeBr}_{6}$.

Kristallisiert sogleich aus und stellt gereinigt schön glänzende, gerade auslöschende, wahrscheinlich rhombische Blättchen dar. Farbe: 4 Orange $p$. Hält sich an der Luft unverändert.

$0.3330 \mathrm{~g}$ Substanz verbrauchten: $25.60 \mathrm{ccm}{ }^{1} / 10^{-n o r m} . \mathrm{AgNO}_{3}$. Berechnet für $\mathrm{C}_{10} \mathrm{H}_{28} \mathrm{~N}_{2} \mathrm{TeBr}_{6}$ :

$$
\begin{array}{cc}
\text { net für } \mathrm{C}_{10} \mathrm{H}_{28} \mathrm{~N}_{2} \mathrm{TeBr}_{8}: & \text { Gefunden: } \\
\mathrm{Br} \quad 61.22 \% & 61.44 \%
\end{array}
$$

7. Di-i-amylammoniumhexabromotellureat, $\left[\left(\mathrm{C}_{5} \mathrm{H}_{11}\right)_{2} \cdot \mathrm{NH}_{2}\right]_{2} \mathrm{TeBr}_{6}$.

Auch hier tritt sogleich ein kristallinischer, orangefarbiger Niederschlag auf, der beim Umkristallisieren merkwürdigerweise zunächst als Öl wiederkommt, sich aber dann schnell in Kristalle verwandelt. Farbe: 4 Orange $o$. - Aus dem Filtrate schieden sich nach mehreren Tagen kleine granatrote Kriställchen, und zwar modellartig ausgebildete und schön klare rhombische Prismen ab. Farbe: 1 Zinnober $k$. Die Präparate weisen beim längeren Aufbewahren an der Luft keinerlei Zersetzungserscheinungen auf.

$0.2376 \mathrm{~g}$ Substanz verbrauchten: $15.45 \mathrm{ccm} 1 / 10^{- \text {norm. }} \mathrm{AgNO}_{3}$. 


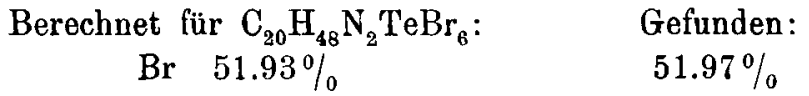

8. Tri-i-amylammoniumhexabromotellureat, $\left[\left(\mathrm{C}_{5} \mathrm{H}_{11}\right)_{3} \cdot \mathrm{NH}\right]_{2} \mathrm{TeBr}_{6}$.

Die Bestandteile liefern beim Vermischen wie bei der entsprechenden Chloroverbindung ein Öl, das nach kurzer Zeit kristallisiert. Man gewinnt bei der Reinigung kleine gelbrote, monokline Kriställchen von schwacher Licht- und Doppelbrechung und einer Auslöschungsschiefe von etwa $40^{\circ}$. Farbe: 5 Orange q. Die Substanz ist luftbeständig.

$0.2030 \mathrm{~g}$ Substanz verbrauchten: $11.55 \mathrm{ccm} 1 / 10^{- \text {norm. }} \mathrm{AgNO}_{3}$. Berechnet für $\mathrm{C}_{30} \mathrm{H}_{68} \mathrm{~N}_{2} \mathrm{TeBr}_{6}$ : Gefunden: Br $45.09 \%$

9. Guanidiniumhexabromotellureat, $\left[\mathrm{HN}: \mathrm{C} \cdot\left(\mathrm{NH}_{2}\right)_{2} \cdot \mathrm{H}\right]_{2} \mathrm{TeCl}_{6}$.

Das Halogenosalz erscheint sogleich als rotgelber kristallinischer Niederschlag, den man bei der Reinigung in Gestalt kleiner, glänzender Nädelchen zurückerhält. Farbe: 4 Orange 0 . - Im Filtrate setzten sich später allmählich derbe, leuchtend rote Kristalle $a b$, die als ganz einfache, nur von der Basis begrenzte, rhombische Prismen erkannt wurden. Farbe: 1 Zinnober c. Die Präparate konnten lange Zeit an der Luft aufbewahrt werden, ohne an Glanz einzubüßen.

$0.1516 \mathrm{~g}$ Substanz verbrauchten: $10.8 \mathrm{ccm} 1 / 10^{1}$-norm. $\mathrm{AgNO}_{3}$.

$$
\begin{array}{cc}
\text { Berechnet für } \mathrm{C}_{2} \mathrm{H}_{12} \mathrm{~N}_{6} \mathrm{TeBr}_{6}: & \text { Gefunden: } \\
\mathrm{Br} \quad 65.94 \% & 65.59 \%
\end{array}
$$

\section{Amidoguanidiniumhexabromotellureat, $\left[\mathrm{HN}: \mathrm{C} . \mathrm{NH}_{2} \cdot \mathrm{NH} \cdot \mathrm{NH}_{3}\right]_{2} \mathrm{TeBr}_{6}$}

Scheidet sich erst nach längerem Stehen der gemischten Lösungen der Bestandteile in meist sehr unregelmäBig ausgebildeten, gerade auslöschenden Kristallen von schwacher Licht- und Doppelbrechung aus. Ganz vereinzelt fanden sich Individuen, die rhombischen Charakter aufwiesen und nur aus Vertikal- und Querprisma bestanden. Farbe: 30 Carmin $i$. Die Präparate trübten sich schon nach verhältnismäBig kurzem Aufbewahren an der Luft.

$0.1756 \mathrm{~g}$ Substanz verbrauchten: $13.9 \mathrm{ccm} 1 / 10^{-n o r m} . \mathrm{AgNO}_{3}$.

Berechnet für $\mathrm{C}_{2} \mathrm{H}_{14} \mathrm{~N}_{8} \mathrm{TeBr}_{6}$ : Gefunden:

$$
\text { Br } 63.33 \%
$$


11. o.Chloraniliniumhexabromotellureat, $\left[\mathrm{C}_{6} \mathrm{H}_{4} \cdot \mathrm{Cl} \cdot \mathrm{NH}_{3}\right]_{2} \mathrm{TeBr}_{6}$.

Man erhält nur eine Abscheidung, wenn man das Reaktionsgemisch vorsichtig etwas einengt. Dann setzen sich nach und nach granatrote, monokline Kriställchen ab, die eine Auslöschungsschiefe von etwa $67^{\circ}$ aufweisen. Farbe: 3 Zinnober $m$. Die Kristalle trüben sich oberflächlich beim Aufbewahren an der Luft.

0.2554 g Substanz verbrauchten: $17.6 \mathrm{ccm} 1 / 10^{-}$-norm. $\mathrm{AgNO}_{3}$

Berechnet für $\mathrm{C}_{12} \mathrm{H}_{14} \mathrm{Cl}_{2} \mathrm{~N}_{2}{ }^{\prime} \mathrm{TeBr}_{6}$ : Gefunden:

Br $55.50 \% \quad 55.06 \%$

12. m-Chloraniliniumhexabromotellureat, $\left[\mathrm{C}_{6} \mathrm{H}_{4}, \mathrm{Cl} . \mathrm{NH}_{3}\right]_{2} \mathrm{TeBr}_{6}$.

Die aus dem sogleich erhaltenen Niederschlage bei der Reinigung gewonnenen Kristalle sind glitzernde, orangerote Blättchen, die sich bei der Betrachtung unter dem Mikroskope als winzige, wahrscheinlich rhombische Prismen mit schiefer Endigung, schiefer Auslöschung, schwacher Lichtbrechung und mäBiger Doppelbrechung erkennen lassen. Farbe: 4 Orange $o$. Das Präparat war an der Luft ziemlich beständig.

$0.3346 \mathrm{~g}$ Substanz verbrauchten: $23.3 \mathrm{ccm} 1 / 10^{\text {-norm. }} \mathrm{AgNO}_{3}$.

Berechnet für $\mathrm{C}_{12} \mathrm{H}_{14} \mathrm{Cl}_{2} \mathrm{~N}_{2} \mathrm{TeBr}_{6}$ : Gefunden:

$$
\text { Br } 55.50 \% \quad 55.65 \%
$$

13. p-Chloraniliniumhexabromotellureat, $\left[\mathrm{C}_{6} \mathrm{H}_{4} \cdot \mathrm{Cl} \cdot \mathrm{NH}_{3}\right]_{2} \mathrm{TeBr}_{6}$.

Auch dieses Halogenosalz scheidet sich direkt kristallinisch, und zwar in Gestalt sehr feiner und winzig kleiner, stark gelbroter Nädelchen aus. In dem Filtrate bildeten sich nach einigen Tagen rotgelbe glänzende Nadeln, die aber keine einheitliche Kristallisation darstellten, sondern sich aus nadelförmigen Kristallen und aus ganz feinen, langen, wahrscheinlich rhombischen Prismen von gerader Auslöschung und schwacher Licht- und Doppelbrechung zusammengesetzt erwiesen. Farbe: 5 Orange $p$. Die Kristalle waren luftbeständig.

$0.1006 \mathrm{~g}$ Substanz verbrauchten $7.0 \mathrm{ccm} 1 / 10$-norm. $\mathrm{AgNO}_{3}$.

Berechnet für $\mathrm{C}_{12} \mathrm{H}_{14} \mathrm{Cl}_{2} \mathrm{~N}_{2} \mathrm{TeBr}_{6}$ : Gefunden:

$\mathrm{Br} 55.50 \%$

14. Dichloranilinium-1, 2, 4-hexabromotellureat, $\left[\mathrm{C}_{6} \mathrm{H}_{3} \cdot \mathrm{Cl}_{2} \cdot \mathrm{NH}_{3}\right]_{2} \mathrm{TeBr}_{6}$.

Erscheint bei der Vermischung der gelösten Bestandteile sogleich als kristallinischer, orangeroter Niederschlag. Farbe: 5 Orange $p$. 
- Das Filtrat liefert nach mehrtägigem Stehen bis zu $1 \mathrm{~cm}$ lange, lebhaft glänzende, rubinrote Kristalle, die feinförmig verwachsen und sehr flächenreich sind und wahrscheinlich dem triklinen Systeme angehören. Farbe: 2 Zinnober $m$. Die Präparate behalten ihren Glanz an der Luft lange Zeit.

$0.0836 \mathrm{~g}$ Substanz verbrauchten: $5.4 \mathrm{ccm} 1 / 10^{- \text {norm. }} \mathrm{AgNO}_{3}$. $0.1428 \mathrm{~g} \quad, \quad \quad, \quad 9.1 \mathrm{ccm} 1 / 10^{-n o r m} . \mathrm{AgNO}_{3}$.

Berechnet für $\mathrm{C}_{12} \mathrm{H}_{12} \mathrm{Cl}_{4} \mathrm{~N}_{2} \mathrm{TeBr}_{8}$ :

Gefunden:

Br $51.40 \%$

15. o-Bromaniliniumhexabromotellureat, $\left[\mathrm{C}_{6} \mathrm{H}_{4} \cdot \mathbf{B r} . \mathrm{NH}_{3}\right]_{2} \mathrm{TeBr}_{6}$.

Die vereinigten Lösungen der Bestandteile liefern bei längerem Stehen bis zu $1 \mathrm{~cm}$ lange dunkelrote, monokline Kristalle (Fig. 7), die von dem Klinopinakoid, der Querfläche und dem Orthodoma begrenzt sind und geringe Auslöschungsschiefe aufweisen. Farbe: 1 Zinnober $k$. Die Kristalle zersetzen sich beim Aufbewahren an der Luft nach einiger Zeit, indem sie farblos und undurchsichtig werden.

$0.0402 \mathrm{~g}$ Substanz verbrauchten : $2.55 \mathrm{ccm}$ $1 / 10$-norm. $\mathrm{AgNO}_{3}$.

Berechnet für $\mathrm{C}_{12} \mathrm{H}_{14} \mathrm{Br}_{2} \mathrm{~N}_{2} \mathrm{TeBr}_{6}$ : Gefunden: $\operatorname{Br} 50.32 \% \quad 50.70 \%$

16. m-Bromaniliniumhexabromotellureat, $\left[\mathrm{C}_{6} \mathrm{H}_{4} \cdot \mathrm{Br} \cdot \mathrm{NH}_{3}\right]_{2} \mathrm{TeBr}_{63}$.

Wenn man die Lösungen der Komponenten miteinander vermischt, beginnt sogleich die Abscheidung eines orangefarbigen, kristallinischen Niederschlages, der beim Umkristallisieren in Form kleiner und feiner Nädelchen wiedergewonnen wird. Farbe: 4 Orange $p$. - Im Filtrate finden sich nach dem Verlaufe von mehreren Tagen braunrote, lebhaft glänzende größere Kristalle vor, die große Ähnlichkeit mit denjenigen des m-Chloraniliniumhexachlorotellureats aufwiesen. Farbe: 1 Zinnober $i$. Die letztgenannte Kristallisation war durchaus luftbeständig.

$0.3904 \mathrm{~g}$ Substanz verbrauchten: $24.6 \mathrm{ccm}{ }^{1} / 10^{-n o r m} . \mathrm{AgNO}_{3}$. 
Berechnet für $\mathrm{C}_{12} \mathrm{H}_{14} \mathrm{Br}_{2} \mathrm{~N}_{2} \mathrm{TeBr}_{6}$ : Br $\quad 50.32 \%$

Gefunden:

$50.36 \%$

17. p-Bromaniliniumhexabromotellureat, $\left[\mathrm{C}_{6} \mathrm{H}_{4}, \mathrm{Br} . \mathrm{NH}_{3}\right]_{2} \mathrm{TeBr}_{6}$.

Nach verhältnismäBig schon kurzer Zeit scheiden die miteinander vereinigten Lösungen der Bestandteile prachtvoll seideglänzende, orangerote Nadeln aus, die sich bei der Betrachtung unter dem Mikroskope als nur von der Basis begrenzte Prismen erkennen lassen. Sie weisen mittlere Licht- und Doppelbrechung auf, löschen gerade aus und sind wahrscheinlich rhombisch. Farbe: 4 Orange $o$. Zersetzungserscheinungen wurden beim Aufbewahren der Präparate an der Luft nicht beobachtet.

$0.0758 \mathrm{~g}$ Substanz verbrauchten: $4.8 \mathrm{ccm} 1 / 10^{-n o r m} . \mathrm{AgNO}_{3}$.

Berechnet für $\mathrm{C}_{18} \mathrm{H}_{14} \mathrm{Br}_{2} \mathrm{~N}_{2} \mathrm{TeBr}_{6}$ :

$\mathrm{Br} \quad 50.32 \%$

Gefunden:

$50.61 \%$

18. m-Nitraniliniumhexabromotellureat, $\left[\mathrm{C}_{6} \mathrm{H}_{4} \cdot \mathrm{NO}_{2} \cdot \mathrm{NH}_{3}\right]_{2} \mathrm{TeBr}_{8}$.

Das Reaktionsgemisch liefert erst nach längerer Zeit eine kristallinische Abscheidung, die aus roten, flächenreichen und wahrscheinlich triklinen Prismen besteht. Farbe: 1 Zinnober $k$. Die Kristalle trüben sich beim Aufbewahren an der Luft oberflächlich.

$0.2274 \mathrm{~g}$ Substanz verbrauchten: $15.35 \mathrm{~cm} 1 / 10^{-n o r m} . \mathrm{AgNO}_{3}$.

Berechnet für $\mathrm{C}_{12} \mathrm{H}_{14} \mathrm{O}_{4} \mathrm{~N}_{4} \mathrm{TeBr}_{6}$ :

$\mathrm{Br} \quad 54.17 \%$

Gefunden:

$53.95 \%$

19. i-Amylaniliniumhexabromotellureat, $\left[\mathrm{C}_{6} \mathrm{H}_{5} \cdot \mathrm{NH}_{2} \cdot \mathrm{C}_{5} \mathrm{H}_{11}\right]_{2} \mathrm{TeBr}_{6}$.

Das Ol, welches man aus dem Reaktionsgemische zunächst erhält, erstarrt schon bald kristallinisch, doch wird auch bei der

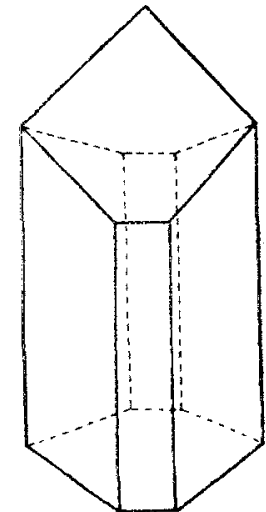

Fig. 8. Reinigung zunächst immer wieder ein Öl abgeschieden. Man kann sich, um Kristalle zu erhalten, nicht anders helfen, als dab man das bei dem Umkristallisieren entstehende Gemenge von Öl und Mutterlauge klärt, indem man bei gewöhnlicher Temperatur viel mehr Alkohol zusetzt, als zur Lösung notwendig wäre. Gibt man die klare Flüssigkeit in eine Porzellanschale und läßt man sie nun lange stehen, so scheiden sich nach und nach hellbräunlichrote, rhombische Kristalle von schwacher Licht- und Doppelbrechung aus. Die so gewonnenen Individuen sind gut 
ausgebildet (Fig. 8) und weisen das Vertikalprisma, die Querfläche, das Querprisma und die Basis auf. Furbe: 5 Orange m. Die Kristalle sind an der Luft nicht lange haltbar; sie zersetzen sich langsam im Laufe einiger Wochen.

0.2268 g Substanz verbrauchten: $14.45 \mathrm{ccm} 1 / 10^{\text {-norm. }} \mathrm{AgNO}_{3}$.

Berechnet für $\mathrm{C}_{22} \mathrm{H}_{36} \mathrm{~N}_{2} \mathrm{TeBr}_{6}$ :

Gefunden:

Br $\quad 51.27 \%$

$50.92 \%$

\section{Benzylaniliniumhexabromotellureat,}

$$
\left[\mathrm{C}_{6} \mathrm{H}_{5} \cdot \mathrm{CH}_{2} \cdot \mathrm{NH}_{2} \cdot \mathrm{C}_{6} \mathrm{H}_{5}\right]_{2} \mathrm{TeBr}_{6} \text {. }
$$

Man erhält bei der Vereinigung der gelösten Bestandteile zunächst ein Öl, das man unter der Mutterlauge in Alkohol löst. Aus der filtrierten Flüssigkeit scheiden sich nach einiger Zeit lebhaft ziegelrote, glänzende, monokline Kristalle von schiefer Auslöschung und schwacher Licht- und Doppelbrechung aus. Sie sind meist sehr einfach ausgebildet und nur von dem Vertikal- und Querprisma begrenzt. Farbe: 3 Zinnober $m$. Eine Zersetzung der Präparate konnte beim Aufbewahren an der Luft nicht konstatiert werden.

$0.2244 \mathrm{~g}$ Substanz verbrauchten: $13.85 \mathrm{ccm} 1 / 10^{- \text {norm. }} \mathrm{AgNO}_{3}$. Berechnet für $\mathrm{C}_{26} \mathrm{H}_{28} \mathrm{~N}_{2} \mathrm{TeBr}_{6}$ :

Br $49.17 \%$

Gefunden:

$49.33 \%$

\section{Methylbenzylaniliniumhexabromotellureat, $\left[\mathrm{C}_{6} \mathrm{H}_{5} \cdot \mathrm{CH}_{2} \cdot \mathrm{NH} \cdot \mathrm{CH}_{3} \cdot \mathrm{C}_{6} \mathrm{H}_{6}\right]_{2} \mathrm{TeBr}_{6}$.}

Aus den gemischten Lösungen der Komponenten scheidet sich ein rotgelbes Öl ab, das ziemlich schnell kristallinisch erstarrt. Erwärmt man den Niederschlag unter der Mutterlauge in Gegenwart einiger Tropfen Alkohol, so erhält man eine klare Flüssigkeit, aus welcher sich beim Abkühlen glänzende Nadeln abscheiden. Es sind kleine Prismen mit schiefer Endigung, schwacher Licht- und Doppelbrechung und einer Auslöschungsschiefe von etwa $35^{\circ}$. Farbe: 5 Orange $p$. Das Präparat war an der Luft lange Zeit beständig.
$0.1500 \mathrm{~g}$ Substanz verbrauchten:
$9.05 \mathrm{ccm} 1 / 10^{- \text {norm. }} \mathrm{AgNO}_{3}$.
$0.0636 \mathrm{~g}$
"
$3.80 \mathrm{ccm} 1 / 10^{-n o r m} . \mathrm{AgNO}_{3}$.

Berechnet für $\mathrm{C}_{28} \mathrm{H}_{32} \mathrm{~N}_{2} \mathrm{TeBr}_{6}$ :

$$
\text { Br } 47.79 \%
$$

Gefunden: 


\section{2. Äthylbenzylaniliniumhexabromotellureat, $\left[\mathrm{C}_{6} \mathrm{H}_{5} \cdot \mathrm{CH}_{2}, \mathrm{NH} \cdot \mathrm{C}_{2} \mathrm{H}_{5} \cdot \mathrm{C}_{6} \mathrm{H}_{5}\right]_{2} \mathrm{TeBr}_{6}$.}

Fällt wie das Chlorosalz zunächst als Öl aus und erstart nach einiger Zeit kristallinisch. Es stellt im gereinigten Zustande kleine, leuchtend rote, stark doppelbrechende Kriställchen dar, die gerade auslöschen und an der Luft keine Zersetzung erleiden. Farbe: 2 Zinnober $m$.

$0.2142 \mathrm{~g}$ Substanz verbrauchten: $12.5 \mathrm{ccm} 1 / 10^{-n o r m} . \mathrm{AgNO}_{3}$. Berechnet für $\mathrm{C}_{30} \mathrm{H}_{36} \mathrm{~N}_{2} \mathrm{TeBr}_{6}$ : $\mathrm{Br} \quad 46.50 \%$ Gefunden: $46.64 \%$

23. Benzalaniliniumhexabromotellureat, $\left[\mathrm{C}_{6} \mathrm{H}_{5} \cdot \mathrm{CH}: \mathrm{NH} . \mathrm{C}_{6} \mathrm{H}_{5}\right]_{2} \mathrm{TeBr}_{6}$.

Wird sogleich kristallinisch gefällt und liefert bei der Reinigung prachtvoll rote, sehr kleine, glänzende Blättchen, die kurze und schmale, dünnprismatische Leistchen von schwacher Licht- und Doppelbrechung darstellen, gerade auslöschen und wohl dem rhombischen System angehören. Farbe: 3 Zinnober o. Die Präparate waren an der Luft beständig.

$0.1302 \mathrm{~g}$ Substanz verbrauchten: $8.0 \mathrm{ccm} 1 / 10$-norm. $\mathrm{AgNO}_{3}$.

Berechnet für $\mathrm{C}_{26} \mathrm{H}_{24} \mathrm{~N}_{2} \mathrm{TeBr}_{6}$ :

Gefunden:

$\mathrm{Br} \quad 49.37 \%$ $49.11 \%$

24. Dimethyl-o-Toluidiniumhexabromotellureat, $\left[\mathrm{C}_{6} \mathrm{H}_{4} \cdot \mathrm{CH}_{3} \cdot \mathrm{N} \cdot\left(\mathrm{CH}_{3}\right)_{2} \cdot \mathrm{H}\right]_{2} \mathrm{TeBr}_{6}$.

Die Komponenten vereinigen sich zu einer roten, öligen Fällung, die bald fest wird und beim Umkristallisieren schön glänzende und lebhaft rote, feine Nädelchen liefert. Farbe: 4 Orange $o$. - Im Filtrate setzen sich nach einiger Zeit hellgranatrote, glänzende Kristalle ab, die modellartig ausgebildete, ganz einfache und an Flächen nur $O P$ und $\infty P$ aufweisende, monokline Prismen sind. Ihr Lichtund Doppelbrechungsvermögen ist schwach, und die Auslöschungsschiefe beträgt etwa $70^{\circ}$. Farbe: 1 Zinnober $k$. Die Kristalle behalten bei längerem Aufbewahren an der Luft ihren Glanz bei. $0.1830 \mathrm{~g}$ Substanz verbrauchten: $12.40 \mathrm{ccm} 1 / 10^{1} \cdot$ norm. $\mathrm{AgNO}_{3}$. Berechnet für $\mathrm{C}_{18} \mathrm{H}_{28} \mathrm{~N}_{2} \mathrm{TeBr}_{6}$ : Gefunden: Br $54.54 \%$ $54.15 \%$ 


\section{Dimethyl-p-Toluidiniumhexabromotellureat, $\left[\mathrm{C}_{6} \mathrm{H}_{4} \cdot \mathrm{CH}_{3} \cdot \mathrm{N} \text {. }\left(\mathrm{CH}_{3}\right)_{2} \cdot \mathrm{H}\right]_{2} \mathrm{TeBr}_{6}$.}

Beim Vermischen der gelösten Bestandteile tritt auch hier zunächst eine ölige Abscheidung auf, die schon nach verhältnismäBig kurzer Zeit erstarrt. Auch beim Umkristallisieren wird unter gewöhnlichen Bedingungen immer nur ein Öl gewonnen, das sich allmählich von selbst, beim Schütteln oder beim Reiben dagegen sogleich in eine feste, aber unreine Masse verwandelt. Man gewinnt ein reines Reaktionsprodukt, wenn man den Niederschlag auflöst, einige Tropfen Alkohol hinzufügt und das Filtrat während des Erkaltens mit einem zurückbehaltenen Kriställchen impft. Dann scheiden sich bald lebhafte glänzende, orangerote Nadeln aus. Farbe: H Orange $p$. - Aus dem Filtrat von dieser Kristallisation setzen sich im Verlaufe mehrerer Tage hell granatrote, gedrungene Nadeln ab, die sich bei der mikroskopischen Untersuchung als gut ausgebildete, rhombische Prismen mit mittlerer Licht- und Doppelbrechung erkennen lassen und gewöhnlich nur die Basis, das Vertikalprisma und die Querfläche aufweisen (Fig. 9). Farbe: 1 Zinnober $i$.

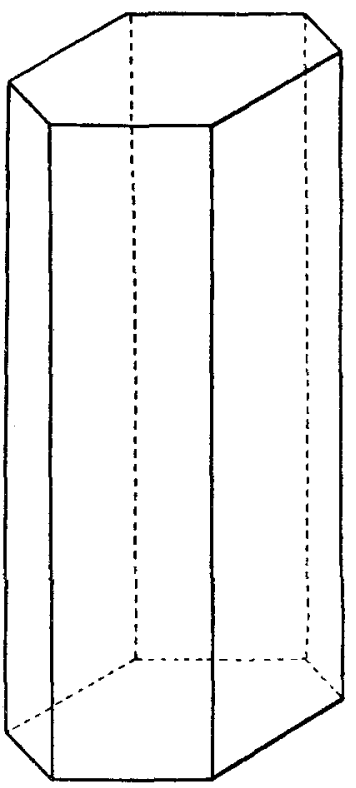

Fig. 9. Die Kristalle erleiden an der Luft keinerlei Zersetzung.

$0.2314 \mathrm{~g}$ Substanz verbrauchten: $15.7 \mathrm{ccm} 1 / 10^{\text {-norm. }} \mathrm{AgNO}_{3}$.

Berechnet für $\mathrm{C}_{18} \mathrm{H}_{28} \mathrm{~N}_{2} \mathrm{TeBr}_{6}$ : Br $54.54 \%$ Gefunden: $54.23 \%$.

26. Pseudocumidiniumhexabromotellureat, $\left[\mathrm{C}_{6} \mathrm{H}_{2} \cdot\left(\mathrm{CH}_{3}\right)_{3} \cdot \mathrm{NH}_{3}\right]_{2} \mathrm{TeBr}_{6}$.

Diese Anlagerungsverbindung setzt sich sogleich als rotgelber, kristallinischer Niederschlag $a b$ und wird bei dem Umkristallisieren in Form stark glänzender, orangefarbiger Blättchen zurückgewonnen. Aus der Mutterlauge scheiden sich allmählich ziemlich breite Nadeln aus, die sich bei der Betrachtung unter dem Mikroskope als monokline, von $P \bar{\infty}, \infty P \bar{\infty}$ und $\infty P \check{\infty}$ begrenzte Prismen erkennen lassen und eine deutliche Spaltbarkeit nach der Längsfläche aufweisen; häufig finden sich Zwillinge nach der Querfläche. Die Lichtbrechung ist schwach, die Doppelbrechung stark, und die Aus- 
löschungsschiefe beträgt etwa $14^{\circ}$. Farbe: 3 Zinnober o. Die Bromoverbindung ist im Gegensatze zu dem entsprechenden Chlorosalze beim Aufbewahren an der Luft ziemlich beständig.

$0.1606 \mathrm{~g}$ Substanz verbrauchten: $11.05 \mathrm{ccm} 1 / 10^{-n o r m} . \mathrm{AgNO}_{3}$. Berechnet für $\mathrm{C}_{18} \mathrm{H}_{28} \mathrm{~N}_{2} \mathrm{TeBr}_{6}$ :

$$
\text { Br } 54.54 \% \quad 54.99 \%
$$

Gefunden:

27. Diphenylammoniumhexabromotellureat, $\left[\left(\mathrm{C}_{6} \mathrm{H}_{5}\right)_{2} \cdot \mathrm{NH}_{2}\right]_{2} \mathrm{TeBr}_{6}$.

Kristallisiert sogleich auch aus stärker verdünnten Lösungen der Bestandteile aus und stellt gereinigt gelbrote, stark licht- und doppelbrechende, wahrscheinlich rhombische, dünne Blättchen dar. Farbe: 4 Orange $m$. Das Präparat ist nicht sehr lange an der Luft beständig; es färbt sich nach einigen Tagen dunkler und ist nach zwei Wochen vollständig zersetzt.

$0.3562 \mathrm{~g}$ Substanz gaben: $0.0466 \mathrm{~g}$ Te.

Berechnet für $\mathrm{C}_{24} \mathrm{H}_{24} \mathrm{~N}_{2} \mathrm{TeBr}_{6}$ : Gefunden:

$$
\text { Br } 13.46 \%
$$

28. Toluylendiammonium-1, 2, 4-hexabromotellureat, $\left[\mathrm{C}_{6} \mathrm{H}_{3} \cdot \mathrm{CH}_{3} \cdot\left(\mathrm{NH}_{3}\right)_{2}\right] \mathrm{TeBr}_{6}$.

Aus den vermischten Lösungen der Bestandteile scheiden sich erst nach einigen Tagen sehr groB und schön ausgebildete rote,

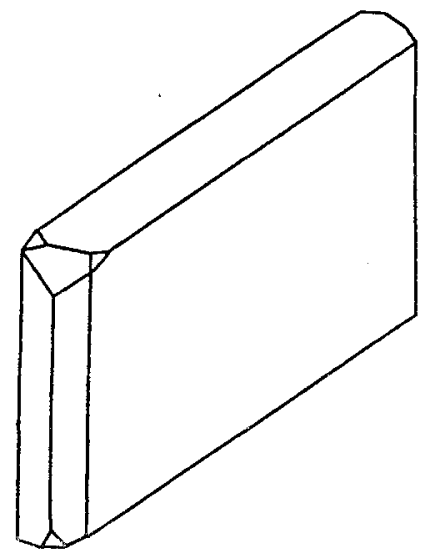

Fig. 10.

glänzende trikline Kristalle aus (Fig. 10). Farbe: 30 Carmin $i$. Das Präparat erwies sich als durchaus luftbeständig und hatte bei mehrwöchentlichem Aufbewahren nichts an Farbe oder Glanz eingebüBt. 
Weitere Beiträge zur Kenntnis der Hexahalogenotellureate. 191

$0.2154 \mathrm{~g}$ Substanz verbrauchten: $17.65 \mathrm{ccm} \mathrm{1/10-norm.} \mathrm{AgNO}_{3}$. Berechnet für $\mathrm{C}_{7} \mathrm{H}_{12} \mathrm{~N}_{2} \mathrm{TeBr}_{6}$ :

Gefunden:

Br $65.59 \%$

$65.49 \%$

29. Toluylendiammonium-1, 3, 4-hexabromotellureat, $\left[\mathrm{C}_{8} \mathrm{H}_{3} \cdot \mathrm{CH}_{3} \cdot\left(\mathrm{NH}_{3}\right)_{2}\right] \mathrm{TeBr}_{6}$.

Man muB das Reaktionsgemisch vorsichtig etwas einengen, um beim Abkühlen die Ausscheidung von orangeroten Kriställchen zu erzielen. Unter dem Mikroskope erkennt man Aggregate von stark licht- und doppelbrechenden, und unregelmäBig ansgebildeten Blättchen, die eine nähere kristallographische Untersuchung nicht gestatteten. Farbe: 2 Zinnober $m$. Änderungen der Farbe oder des Glanzes wurden beim Aufbewahren dieser Substanz an der Luft nicht bemerkt.

$0.4446 \mathrm{~g}$ Substanz gaben: $0.0764 \mathrm{~g}$ Te.

$\begin{array}{cc}\text { Berechnet für } \mathrm{C}_{7} \mathrm{H}_{12} \mathrm{~N}_{2} \mathrm{TeBr}_{6}: & \text { Gefunden: } \\ \mathrm{Te} 17.44 \% & 17.18 \%\end{array}$

30. o-Phenetidiniumhexabromotellureat, $\left[\mathrm{C}_{6} \mathrm{H}_{4} \cdot \mathbf{0} \cdot \mathrm{C}_{2} \mathrm{H}_{5} \cdot \mathrm{NH}_{3}\right]_{2} \mathrm{TeBr}_{6}$.

Die gelösten Bestandteile liefern beim Vermischen einen orangefarbigen kristallinischen Niederschlag, der sich aus gut ausgebildeten,

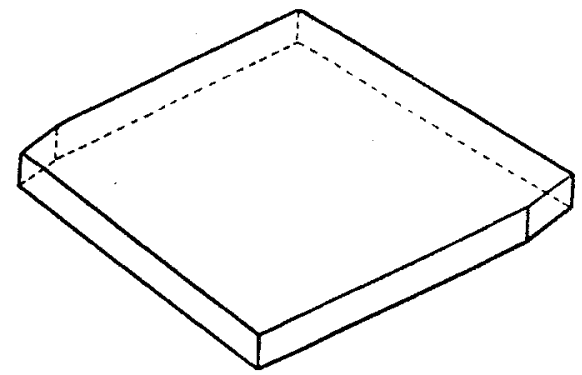

Fig. 11.

sechseckigen, schwach licht- und stark doppelbrechenden, glänzenden, rhombischen Blättchen zusammengesetzt erwies (Fig. 11). Beobachtet wurden Basis, Vertikalprisma und Längsfläche. Farbe: 4 Orange q. Das Präparat weist beim Aufbewahren an der Luft keine Zersetzungserscheinungen auf.

$0.3650 \mathrm{~g}$ Substanz gaben: $0.0518 \mathrm{~g}$ Te.

Berechnet für $\mathrm{C}_{16} \mathrm{H}_{24} \mathrm{O}_{2} \mathrm{~N}_{2} \mathrm{TeBr}_{6}$ : Gefunden:

Te $14.43 \%$ 


\section{1. p-Phenetidiniumhexabromotellureat, $\left[\mathrm{C}_{6} \mathrm{H}_{4} \cdot 0 \cdot \mathrm{C}_{2} \mathrm{H}_{5} \cdot \mathrm{NH}_{3}\right]_{2} \mathrm{TeBr}_{6}$.}

Auch hier tritt sogleich ein kristallinischer Niederschlag auf. Man beobachtet schwach licht. und doppelbrechende, rhombische, von der Basis, dem Vertikalprisma und der Querfläche begrenzte Kriställchen. Farbe: 4 Orange $o$. Hält sich an der Luft unzersetzt.

0.1260 g Substanz gaben: 0.0178 g Te.

$$
\begin{array}{cc}
\text { Berechnet für } \mathrm{C}_{16} \mathrm{H}_{24} \mathrm{O}_{2} \mathrm{~N}_{2} \mathrm{TeBr}_{6}: & \text { Gefunden: } \\
\text { Te } 14.43 \% & 14.13 \%
\end{array}
$$

32. $\beta$-Picoliniumhexabromotellureat, $\left[\mathrm{C}_{5} \mathrm{H}_{4} \cdot \mathrm{CH}_{3} \cdot \mathrm{NH}\right]_{2} \mathrm{TeBr}_{6}$.

Das Halogenosalz erscheint sogleich als gelblichroter, kristallinischer Niederschlag, der zunächst wohl Neigung zur öligen Abscheidung besitzt, aber bald vollständig fest wird. Er stellt im gereinigten Zustande prachtvoll rotbraune, wahrscheinlich monokline Kriställchen von schwacher Licht- und Doppelbrechung dar. Farbe: 8 Orange $r$. Die Substanz hält sich an der Luft lange, ohne Spuren einer Zersetzung aufzuweisen.

$0.2066 \mathrm{~g}$ Substanz verbrauchten: $15.65 \mathrm{ccm} 1 / 10^{- \text {norm. }} \mathrm{AgNO}_{3}$.

Berechnet für $\mathrm{C}_{12} \mathrm{H}_{16} \mathrm{~N}_{2} \mathrm{TeBr}_{6}$ : Gefunden:

$$
\text { Br } 60.30 \% \quad 60.54 \%
$$

33. Lutidiniumhexabromotellureat, $\left[\mathrm{C}_{5} \mathrm{H}_{3} \cdot \mathrm{N} \cdot\left(\mathrm{CH}_{3}\right)_{2} \cdot \mathrm{H}\right]_{2} \mathrm{TeBr}_{6}{ }^{1}$

Im Gegensatze zu der entsprechenden Chloroverbindung wird hier zunächst ein Öl abgeschieden, das bei der Reinigung - man setzt vorteilhaft einige Tropfen Alkohol hinzu - in Gestalt von wahrscheinlich rhombischen Prismen mit aufgesetzter Pyramide wiedergewonnen wird. An den Kristallen wurde schwache Lichtbrechung und starke Doppelbrechung konstatiert. Farbe: 4 Orange $m$. Das Präparat war luftbeständig.

$0.2235 \mathrm{~g}$ Substanz verbrauchten: $16.3 \mathrm{ccm} 1 / 10^{-n o r m} . \mathrm{AgNO}_{3}$.

Berechnet für $\mathrm{C}_{14} \mathrm{H}_{20} \mathrm{~N}_{2} \mathrm{TeBr}_{6}$ : Gefunden:

$$
\operatorname{Br} 58.25 \% \quad 58.29 \%
$$

34. Kollidiniumhexabromotellureat, $\left[\mathrm{C}_{5} \mathrm{H}_{2} \cdot\left(\mathrm{CH}_{3}\right)_{9} \cdot \mathrm{NH}_{2} \mathrm{TeBr}_{6} \cdot{ }^{2}\right.$

Scheidet sich sogleich in Gestalt eines roten Öles ab, das auf Zusatz von konzentrierter Bromwasserstoffsäure kristallinisch erstarrt.

'Ausgangsmaierial: Lutidin käuflich.

2 Ausgangsmaterial: Kollidin aus Aldehyd. 
Man beobachtet bei der Reinigung, daß das Halogenosalz immer wieder ölig ausfällt und erst durch starkes Schütteln des Reaktionsgemisches fest wird. Farbe: 4 Orange $m$. - Ein reines Produkt wurde aus dem Filtrat gewonnen, das nach einigen Tagen hellrote glänzende Kriställchen ausschied. Es sind an der Basis abgestumpfte rhombische Pyramiden von schwacher Lichtbrechung und ziemlich starker Doppelbrechung. Farbe: 3 Zinnober $o$. Die Kristalle büßten nichts an Glanz ein, wenn sie längere Zeit an der Luft aufgehoben wurden.

$0.3020 \mathrm{~g}$ Substanz verbrauchten: $21.3 \mathrm{ccm} 1 / 10^{-n o r m} . \mathrm{AgNO}_{3}$. $0.2578 \mathrm{~g} \quad, \quad 18.2 \mathrm{ccm} 1 / 10^{\text {-norm. }} \mathrm{AgNO}_{3}$. Berechnet für $\mathrm{C}_{16} \mathrm{H}_{24} \mathrm{~N}_{2} \mathrm{TeBr}_{6}$ : Gefunden: Br $56.33 \% \quad 56.37 ; 56.42 \%$

35. Piperidiniumhexabromotellureat, $\left[\mathrm{C}_{5} \mathrm{H}_{10} \cdot \mathrm{NH}_{2}\right]_{2} \mathrm{TeBr}_{6}$.

Beim Vermischen der gelösten Bestandteile kristallisieren sogleich orangefarbige, prachtroll glitzernde, kleine Kriställchen aus.

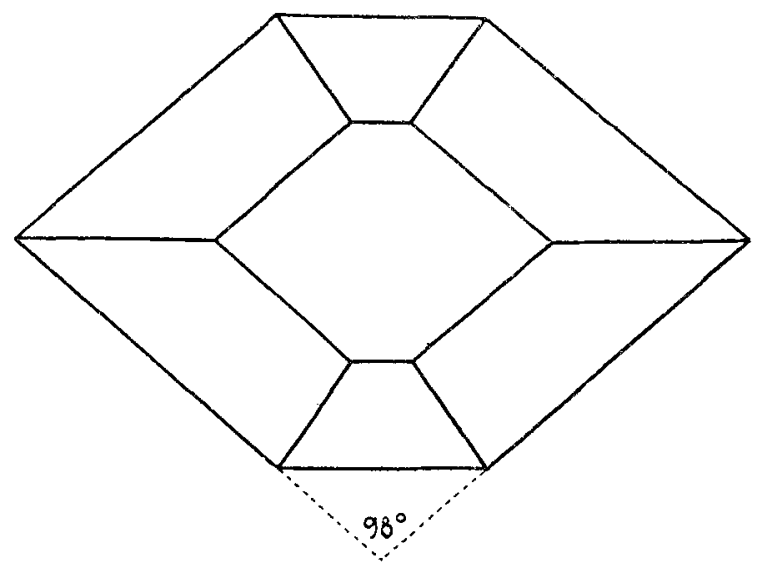

Fig. 12.

Farbe: 4 Orange o. Im Filtrate erscheinen nach einiger Zeit gut und gleichmäBig ausgebildete, etwa $0.5 \mathrm{~mm}$ grobe, rote, rhombische Kristalle, die von dem Querprisma, einer Pyramide und der Basis begrenzt sind (Fig. 12), und schwache Lichtbrechung sowie starke Doppelbrechung aufweisen. Farbe: 1 Zinnober $k$. Die Präparate waren luftbeständig.

$0.3134 \mathrm{~g}$ Substanz verbrauchten: $24.05 \mathrm{ccm} 1 / 10^{\text {-norm. }} \mathrm{AgNO}_{3}$. 
Berechnet für $\mathrm{C}_{10} \mathrm{H}_{24} \mathrm{~N}_{2} \mathrm{TeBr}_{6}$ :

Gefunden:

$\mathrm{Br} \quad 61.54 \%$

$61.33 \%$

36. Benzoylpiperidiniumhexabromotellureat, $\left[\mathrm{C}_{5} \mathrm{H}_{10} \text {. NH.CO. } \mathrm{C}_{6} \mathrm{H}_{5}\right]_{2} \mathrm{TeBr}_{6}$.

Diese Anlagerungsverbindung bildet hellrote, große und breite Nadeln, die dem monoklinen System angehören und nur von $\infty P \infty$, $\infty P$ und $O P$ begrenzt sind; die Auslöschungsschiefe beträgt etwa $60^{\circ}$. Farbe: 3 Zinnober $m$. Das Bromosalz erlitt auch bei längerem Liegen an der Luft keine Zersetzung.

$0.1629 \mathrm{~g}$ Substanz verbrauchten: $9.65 \mathrm{ccm} 1 / 10^{-n o r m} . \mathrm{AgNO}_{3}$.

Berechnet für $\mathrm{C}_{24} \mathrm{H}_{32} \mathrm{O}_{2} \mathrm{~N}_{2} \mathrm{TeBr}_{6}$ :

$\operatorname{Br} \quad 48.57 \%$

Gefunden:

$47.35 \%$

37. i-Chinoliniumhexabromotellareat, $\left[\mathrm{C}_{9} \mathrm{H}_{7} \cdot \mathrm{NH}\right]_{2} \mathrm{TeBr}_{6}$.

Kristallisiert sogleich aus und wird bei der Reinigung in schön seideglänzenden, orangefarbigen Nadeln zurückgewonnen. Es sind winzig feine, wohl monokline Prismen von schwacher Lichtbrechung, mäBiger Doppelbrechung und einer Auslöschungsschiefe von etwa $37^{\circ}$. Farbe: 2 Zinnober $m$. Das Bromosalz ist luftbeständig.

$0.1298 \mathrm{~g}$ Substanz verbrauchten: $9.0 \mathrm{ccm} 1 / 10^{-}$-norm. $\mathrm{AgNO}_{3}$. $0.1980 \mathrm{~g} \quad$ " $13.65 \mathrm{ccm} 1 / 10^{-n o r m} . \mathrm{AgNO}_{3}$.

Berechnet für $\mathrm{C}_{18} \mathrm{H}_{16} \mathrm{~N}_{2} \mathrm{TeBr}_{6}$ : Gefunden:
Br $55.30 \%$
$55.3755 .10 \%$

38. Benzylmethylammoniumhexabromotellureat,

$$
\left[\mathrm{C}_{6} \mathrm{H}_{5} \cdot \mathrm{CH}_{2} \cdot \mathrm{CH}_{3} \cdot \mathrm{NH}_{2}\right]_{2} \mathrm{TeBr}_{6} \text {. }
$$

Aus den vermischten Lösungen der Komponenten fällt sogleich ein kristallinischer Niederschlag aus, der bei der Reinigung in Form von seideglänzenden, orangeroten Leistchen zurückerhalten wird. Man erkennt unter dem Mikroskop winzige Prismen von schwacher Lichtbrechung, starker Doppelbrechung und einer Auslöschnngsschiefe von $60^{\circ}$. Farbe: 4 Orange $q$. Die Substanz veränderte sich beim Liegen an der Luft nicht.

$0.1444 \mathrm{~g}$ Substanz verbrauchten: $10.1 \mathrm{ccm} 1 / 10$-norm. $\mathrm{AgNO}_{3}$.

Berechnet für $\mathrm{C}_{16} \mathrm{H}_{24} \mathrm{~N}_{2} \mathrm{TeBr}_{6}$ :

Gefunden:

Br $56.33 \%$

$55.90 \%$ 
39. Benzalmethylammoniumhexabromotellureat, $\left[\mathrm{C}_{6} \mathrm{H}_{5} \text {. CH: } \mathrm{NH} . \mathrm{CH}_{3}\right]_{2} \mathrm{TeBr}_{6}$.

Setzt sich sogleich als körniger Niederschlag $a b$ und bildet (wahrscheinlich rhombische) gerade auslöschende orangefarbige Prismen von mittlerer Licht- und Doppelbrechung. Farbe: 4 Orange $o$. Zersetzt sich an der Luft nicht.

$0.2780 \mathrm{~g}$ Substanz verbrauchten: $19.9 \mathrm{~cm} 1 / 10^{-n o r m} . \mathrm{AgNO}_{3}$.

Berechnet für $\mathrm{C}_{16} \mathrm{H}_{20} \mathrm{~N}_{2} \mathrm{TeBr}_{6}$ : Gefunden:

Br $56.60 \%$ $57.21 \%$

\section{Benzaläthylammoniumhexabromotellureat,} $\left[\mathrm{C}_{6} \mathrm{H}_{5} . \mathrm{CH}: \mathrm{NH} . \mathrm{C}_{2} \mathrm{H}_{5}\right]_{2} \mathrm{TeBr}_{6}$.

Wird zunächst als Öl erhalten, das schnell erstarrt, und liegt nach der Reinigung in schön glänzenden, orangeroten Blättchen vor. Aus dem Filtrate schieden sich nach einiger Zeit kleine gelbrote Kriställchen ans, die mittlere Licht- sowie Doppelbrechung und eine Auslöschungsschiefe von etwa $44^{\circ}$ aufweisen und wahrscheinlich monoklin sind. Farbe: 5 Orange $q$. Das Präparat erlitt an der Luft keinerlei Zersetzung.

$0.2946 \mathrm{~g}$ Substanz verbrauchten: $20.3 \mathrm{ccm} 1 / 10^{-n o r m} . \mathrm{AgNO}_{2}$.

Berechnet für $\mathrm{C}_{18} \mathrm{H}_{24} \mathrm{~N}_{2} \mathrm{TeBr}_{6}$ :

$$
\mathrm{Br} 54.79 \% \quad 55.07 \%
$$

Stuttgart, Laboratorium für Elektrochemie und 'lechnische Chemie der Technischen Hochschule, und Würnburg.

Bei der Redaktion eingegangen am 9. Februar 1914. 